\title{
Competency in Acute Resuscitation Through Successive Simulation (CARTSS): a mentor based, near-peer learning initiative
}

\author{
John R. O'Leary, BScH, MSc*; Natasha L. Goumeniouk, BSc*; Alexander S. Cormier*; \\ Daniel J. Potter, BSc*; Filip Gilic, MD $^{*}{ }^{\dagger}$; Erin E. Brennan, MD, MMEd ${ }^{\ddagger}$
}

\section{BACKGROUND}

Current life support training standards involve participating in a two-day training course that certifies providers for a two-year period. The course consists of a didactic component and some hands-on practice with cardiopulmonary resuscitation (CPR) manikins. Studies have shown that the quality of training at these sessions can vary greatly. ${ }^{1}$ Furthermore, skill testing only three to six months after course completion has shown that participants perform chest compressions well below recommended guidelines, with ineffective rates and shallow compression depths, resulting in the potential for poor perfusion. ${ }^{2,3}$ For this reason, some groups have taken a different approach to the traditional biennial certification model. This new approach consists of participation in shorter and more frequent training sessions with real-time feedback on skill performance. Retention rates for these short course high-frequency programs have been variable, and the ideal frequency at which to participate in a training session has yet to be established. However, many of these programs are seeing positive results with this increased frequency approach to skill cultivation. ${ }^{3-5}$

\section{RATIONALE}

To provide quality, high-frequency training, we created a mentorship-based, near-peer learning program. Our program looked at using this approach to improve compression efficacy and skill retention for medical trainees. Secondary skill training included teaching basic airway management, proper bag-valve mask technique, defibrillator setup, safety, and closed-loop communication and improving overall confidence in resuscitation skills for medical students. Any one of these components could be stand-alone training sessions; however, this model allowed us to look at the effects of the training format, frequency, and content as we further developed the program.

To use our simulation lab time effectively, we held basic science classroom-based learning sessions and developed online video resources to help prepare participants before they arrived for simulation training. In all, participants committed roughly four hours to the program over the course of the academic year, including at-home training through online modules. A key goal of the project was to keep the time commitment as minimal as possible while still providing a program that optimized learning and skill retention.

Another goal of our program was to expose students to the simulation lab early in medical school to increase their comfort with this form of training. While the main goal of the program was improvement in fundamental resuscitation skills, we hoped to provide participants with the experience of being in full simulations in which they had to act as a team member or leader. Because of its resourceintensive nature and high-demand, medical students often have minimal exposure to this kind of simulation training during their first two years of education.

\section{DESCRIPTION}

We developed an educational intervention centred around the concepts of higher-frequency peer-led training sessions. Skills were practised using both

From the *School of Medicine, Queen's University, Kingston, ON; †Queen's Department of Family Medicine, Queens University, Kingston, ON; and $¥$ Department of Emergency Medicine, Queen's University, Kingston, ON.

Correspondence to: Natasha Goumeniouk, Department of Emergency Medicine, Kingston Health Sciences Centre, 76 Stuart St., Kingston, ON K7L 2V7, Canada; E-mail: ngoumeniouk@ qmed.ca 
high- and low-fidelity simulation manikins that provide real-time feedback on operator technique. Instructor feedback was also provided by upper-year medical students and staff physicians. We called this program Competent Acute Resuscitation Through Successive Simulation or CARTSS.

The basic components taught to the first-year participants by second-year instructors included high-quality CPR, bag-valve mask ventilation, and defibrillator initialization and basic operation. The preliminary instruction was provided in online videos demonstrating each component individually, as well as in a coordinated resuscitation scenario. The participants engaged in 45-minute in-person training sessions, during which small groups of three to five participants were mentored by upper-year instructors who had previously been trained in and practised the fundamental skills. During the training sessions, learners first practised each skill individually, before each taking a turn leading a team in a simulated resuscitation (Figure 1). Second-year instructors were trained by staff physicians, residents, and clerks on more advanced resuscitation roles, so they could provide effective feedback to the incoming participants. All training was supervised by a staff emergency physician to ensure quality; however, the goal was for near-peer mentors to provide direct small-group instruction. Students of all levels also received numeric CPR metrics and individualized verbal feedback on the efficacy of their resuscitation skills during and after the sessions.

\section{DISCUSSION}

We found mentorship-based near-peer instruction was the key to being able to provide frequent and effective training sessions to a large number of participants. In this system, two staff physicians trained a smaller number of more senior medical students to act as instructors. This method allowed for direct small-group teaching and individualized feedback for a greater number of students. These student instructors were coordinated by a smaller group of student coordinators who were tasked with course communication, research question development, and curriculum design for each year of the program.

As this was the first year of development of the project, student instructors were selected from senioryear medical students based on their interest in involvement. However, moving forward, our student instructors will be selected from students who were involved in the program the year before and showed interest in remaining as educators. The coordinators will be selected from previous student instructors and
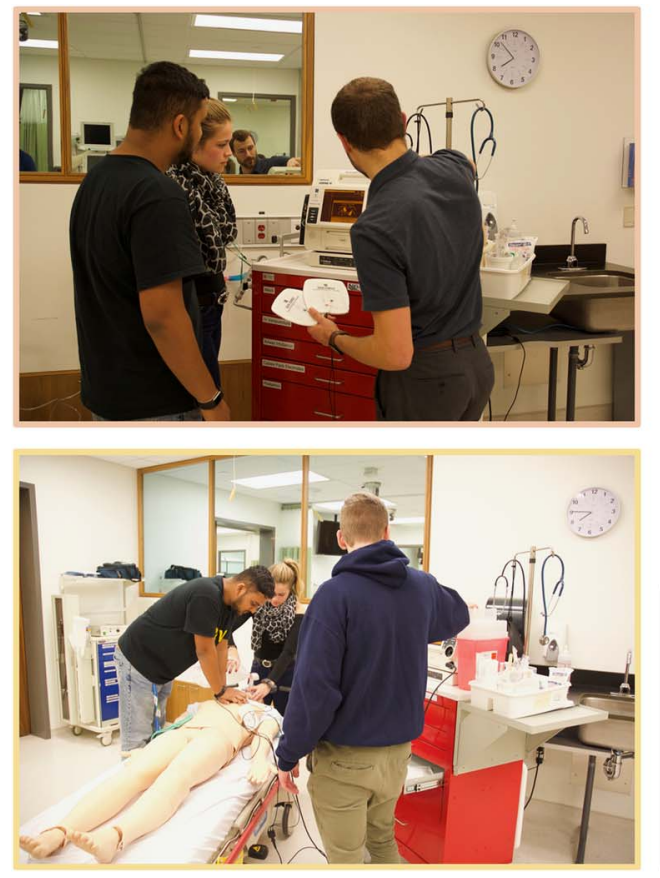

Figure 1. A representation of the layout of the Queen's Clinical Simulation Centre labs used for the training program. Arrows indicate the flow of students (in groups of three to five) through the three different core competency stations and on the final full simulation. All stations are monitored by staff through the central control room.
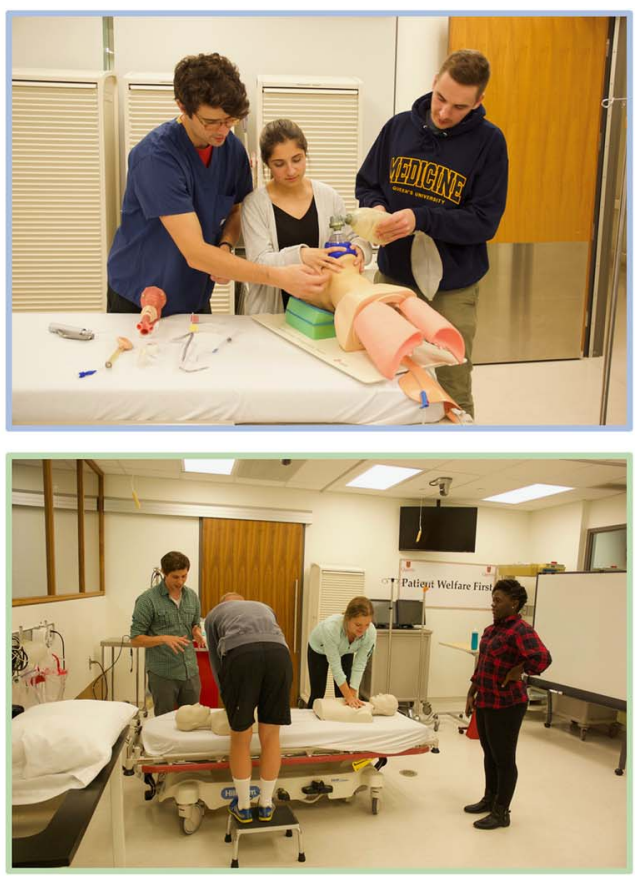
given the freedom to refine and develop the program further. The intention is to build upon the foundation of the core curriculum and develop new research questions on an annual basis.

\section{SUMMARY}

The focus of this innovation is to provide students in the Queen's medicine program access to a highfrequency and high-fidelity simulation of acute resuscitation. We hope to ensure they develop the basic resuscitation skills they may be asked to perform once they begin clerkship. By providing consistent practice in high-stress situations through low-risk simulation, we hope they will be better equipped to manage acute care situations that may arise throughout their medical education. The program is maintained and expanded through successive levels of student mentorship, providing medical trainees an early introduction to the formal and informal teaching mechanisms that they will encounter during their lifelong educational career. Through a mentorship-based and high-frequency training approach, CARTSS prepares students to understand and participate in resuscitation scenarios that they may encounter in a hospital setting.
Keywords: simulation, resuscitation, medical education

Acknowledgements: Thank you to the Queen's Clinical Simulation Centre staff for their support in founding and continuing this program.

Competing interests: This work was supported by the Kingston Resuscitation Institute, the Queen's Aesculapian Society Initiative Award, and the Ontario Medical Student Association's Innovator Grant. No competing interests are declared.

\section{REFERENCES}

1. Kaye W, Rallis SF, Mancini ME, et al. The problem of poor retention of cardiopulmonary resuscitation skills may lie with the instructor, not the learner or the curriculum. Resuscitation 1991;21(1):67-87.

2. Kaye $W$, Mancini ME. Retention of cardiopulmonary resuscitation skills by physicians, registered nurses, and the general public. Crit Care Med 1986;14(7):620-2.

3. Niles D, Sutton RM, Donoghue A, et al. "Rolling Refreshers": a novel approach to maintain CPR psychomotor skill competence. Resuscitation 2009;80(8):909-12.

4. Cheng A, Brown LL, Duff JP, et al. Improving cardiopulmonary resuscitation with a CPR feedback device and refresher simulations (CPR CARES Study): a randomized clinical trial. 7AMA Pediatr 2015;169(2):137-44.

5. Niles DE, Nishisaki A, Sutton RM, et al. Improved retention of chest compression psychomotor skills with brief "Rolling Refresher" training. Simul Healthc 2017;12(4):213-9. 